\title{
TRAUMATISMO RAQUIMEDULAR POR FERIMENTO DE PROJÉTIL DE ARMA DE FOGO: AVALIAÇÃO EPIDEMIOLÓGICA
}

\author{
SPINAL CORD TRAUMA BY A FIREARM PROJECTILE: EPIDEMIOLOGICAL ASSESSMENT \\ TRAUMATISMO RAQUIMEDULAR POR HERIDA DE UN PROYECTIL DE ARMA DE FUEGO: \\ LA EVALUACIÓN EPIDEMIOLÓGICA
}

Francisco Alves de Araújo Júnior ${ }^{1}$, Cristina Belotserkovets Heinrich'1 Marcelo lemos Vieira Cunha², Denildo César Amaral Veríssimo², Roberta Rehder², Camila Araújo Scharf Pinto ${ }^{3}$, Samir Ale Bark ${ }^{4}$, Luiz Alencar Birrium Borba ${ }^{5}$

\begin{abstract}
RESUMO
Objetivo: Avaliar a incidência de trauma raquimedular causado por projétil de arma de fogo nos pacientes atendidos no pronto socorro de um hospital de Curitiba (PR). Métodos: Estudo retrospectivo, realizado no período de fevereiro de 2005 a julho de 2008 , incluindo todos os pacientes, vitimas de lesão na coluna vertebral causada por projétil de arma de fogo $(n=54)$. Resultados: A grande maioria dos pacientes acometidos é do sexo masculino e em idade produtiva, com média de 27,18 anos. O segmento mais afetado foi a coluna torácica, com ferimentos transfixantes e lesão ASIA A (lesão completa). A maioria dos casos foi tratada de forma conservadora. O período da semana em que houve maior incidência foi o fim e semana, com predomínio do sábado e da sexta-feira. O motivo mais comum foi assalto. Conclusão: São lesões com alto impacto no custo de saúde pública e de previdência, uma vez que são lesões de alta taxa de morbidade e acometimento predominante de pessoas em faixa etária produtiva.
\end{abstract}

Descritores: Coluna vertebral; Traumatismo da coluna vertebral/epidemiologia; Ferimentos por arma de fogo.

\begin{abstract}
Objective: To evaluate the incidence of spinal cord injury caused by firearm projectile in patients admitted to the emergency room of a hospital in Curitiba (PR). Methods: This retrospective study from February 2005 to July 2008 including all victims of spinal injury caused by firearm projectile $(n=54)$. The data were analyzed descriptively. Results: The vast majority of patients are male and of working age, with an average of 27.18 years. The thoracic spine was the most affected segment, with transfixing injury and ASIA A (complete lesion). Most cases were treated conservatively. The period of the week where there was a higher incidence was the weekend, mostly on Saturday and Friday. The most common reason was armed robbery. Conclusion: These are lesions with a high impact on the cost of public health and social security, as are injuries that result in high morbidity and involve predominantly people in the productive age group.
\end{abstract}

Keywords: Spine; Spinal trauma/epidemiology; injuries with firearms.

\section{RESUMEN}

Objetivo: Evaluar la incidencia del traumatismo raquimedular causado por proyectil de arma de fuego en pacientes atendidos en la sala de emergencias de un hospital en Curitiba (Paraná). Métodos: Estudio retrospectivo, realizado en el período de febrero 2005 a julio de 2008, incluyendo a todos los pacientes víctimas de lesión medular en la columna vertebral causada por un proyectil de arma de fuego ( $n=54$ pacientes). Resultados: La gran mayoría de los pacientes son varones y en edad de trabajar, con un promedio de 27,18 años. El sector más afectado ha sido la columna torácica, con heridas transfixiantes y ASIA A (lesión medular completa). La mayoría de los casos fue tratada de forma conservadora. El período de la semana, donde había una mayor incidencia fue el fin de semana, con predominio del sábado y el viernes. La razón más frecuente fue por asalto. Conclusión: Se trata de lesiones con un alto impacto en el costo de la salud pública y la Seguridad Social, considerando que son lesiones con una alta tasa de morbilidad y afectan predominante a personas en el grupo de edad productiva.

Descriptores: Columna vertebral; Traumatismos vertebrales/epidemiologia; Heridas por arma de fuego.

\section{INTRODUÇÃO}

O aumento da violência urbana é uma situação que pode ser notada pelas notícias diárias. As lesões provocadas pelas armas de fogo correspondem à segunda causa de lesões medulares, superada apenas pelos acidentes automobilísticos ${ }^{1-3}$

O segmento torácico da coluna vertebral é o mais frequentemente atingido devido ao seu maior comprimento. As lesões ao nível da coluna cervical são as mais graves pela seqüela neurológica que produzem. Os paciente jovens e do sexo masculino são os mais afetados e, os acidentes, acontecem predominantemente nos finais de semana ${ }^{1,4-6}$. Na grande maioria das vezes, são acompanhados de déficit neurológico devido à lesão medular ou radicu$\operatorname{lar}^{7}$. O grau de lesão tecidual depende da energia transmitida pelo projétil aos tecidos, no momento do trauma. Essa energia varia de acordo com a massa do projétil e com o quadrado da velocidade atingida pelo mesmo. Quando um projétil atinge a coluna, provoca lesões primárias devido ao trauma mecânico direto e trauma térmico sobre estruturas neurais e vasculares ${ }^{7-9}$. Posteriormente, podem ocorrer lesões secundárias seja por isquemia ou hiperemia.

1. Médico Neurocirurgião formado pelo Serviço de Residência Médica de Neurocirurgia do Hospital Universitário Evangélico de Curitiba (HUEC) - Curitiba, PR, Brasil.

2. Médicos Residentes do Serviço de Neurocirurgia do HUEC - Curitiba, PR, Brasil.

3. Acadêmica do Curso de Medicina da Faculdade Evangélica do Paraná (FEPAR) - Curitiba, PR, Brasil.

4. Médico Neurocirurgião preceptor da Residência Médica em Neurocirurgia do HUEC e Professor da Disciplina de Neuroanatomia do Curso de Medicina da FEPAR - Curitiba, PR, Brasil.

5. Mestre em Neurocirurgia pela Unifesp e Doutor em Cirurgia pela FMUSP - Ribeirão Preto, Médico Neurocirurgião coordenador do Serviço de Neurocirurgia do HUEC ) - Curitiba, PR, Brasil. 
Atualmente nos Estados Unidos, cerca de 183 a 203 mil pessoas apresentam lesões medulares e o gasto com estes pacientes varia de acordo com a idade do paciente e a gravidade da lesão. Um paciente de 25 anos com quadriplegia alta custa cerca de US\$ 1.350.000,00 enquanto que uma pessoa de 50 anos com paraplegia custa algo em torno de US\$326.000,007

O objetivo desse estudo é avaliar a incidência destas lesões nos pacientes atendidos no pronto socorro de um hospital de referência em traumatologia em Curitiba, PR.

\section{MÉTODOS}

Foram coletados dados retrospectivamente de prontuários de pacientes com lesões vertebrais por arma de fogo, que foram atendidos no pronto socorro do Hospital Universitário Evangélico de Curitiba. O período avaliado foi de fevereiro de 2005 a julho de 2008. Dados como idade, sexo, nível acometido (cervical, torácico, lombar ou sacral), grau da lesão medular (ASIA A, B, C, D ou E), tempo de internamento, dia da semana do trauma, causa do ferimento, mortalidade, tipo de tratamento (conservador ou cirúrgico) e tipo de ferimento (intra ósseo, transfixante ou intracanal) foram coletados. Os dados foram anotados em protocolo do trabalho e analisados de modo descritivo.

\section{RESULTADOS}

Dos 54 pacientes, 49 eram do sexo masculino $(90,7 \%)$ e cinco do sexo feminino $(9,3 \%)$. A idade média foi de 27,18 anos, variando entre 15 a 55 anos. Estratificando-se a faixa etária (Figura 1), encontramos 11 pacientes de 15 a 20 anos (20,3\%; 26 pacientes entre 21-30 anos (48,3\%), nove pacientes entre $31-40$ anos $(16,6 \%)$, seis entre $41-50$ anos $(11,1 \%)$ e dois entre $51-55$ anos $(3,7 \%)$.

A causa mais frequente dos disparos foi por assalto em $40 \mathrm{ca}$ sos (74\%), seguido de briga em 11 casos (20,4\%). Em três casos não foi identificada a causa $(5,6 \%)$ devido os pacientes terem sido encontrados inconscientes em via pública.

O período da semana (Figura 2) com maior incidência de caso foi o sábado com 16 casos (29,6\%) seguido da sexta feira com 15 casos (26\%).

Os níveis mais cometidos (Figura 3) foram o torácico com 23 casos (42,6\%), seguido pelo cervical com 20 casos $(37,1 \%)$, lombar com nove casos $(16,6 \%)$ e sacral com dois casos $(3,7 \%)$.

O tipo de ferimento (Figura 4) foi dividido entre lesões com o projétil alojado na vértebra em 20 casos (37\%), com o projétil dentro do canal vertebral em oito casos (15\%) e com lesões transfixantes em 26 casos (48\%).

O grau de lesão neurológica (Figura 5) deu-se no seguinte padrão: ASIA A (lesão completa) 24 casos (44,4\%), ASIA B em um caso (1,8\%), ASIA C em quatro casos (7,4\%), ASIA D em cinco casos $(9,4 \%)$ e ASIA E (assintomáticos) em 20 casos (37\%).

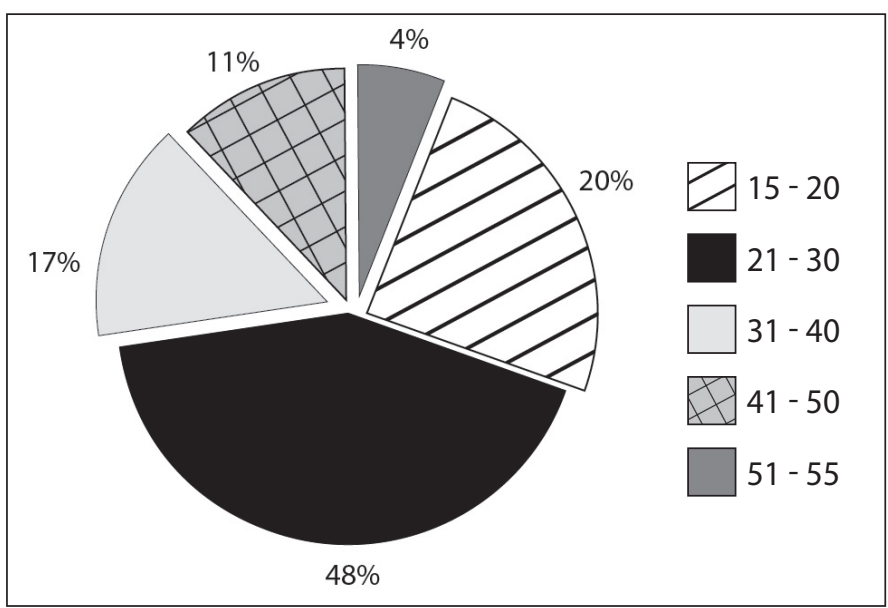

Figura 1. Faixa-etária dos pacientes vítimas de ferimento por projétil de arama de fogo (FPAF) na coluna.

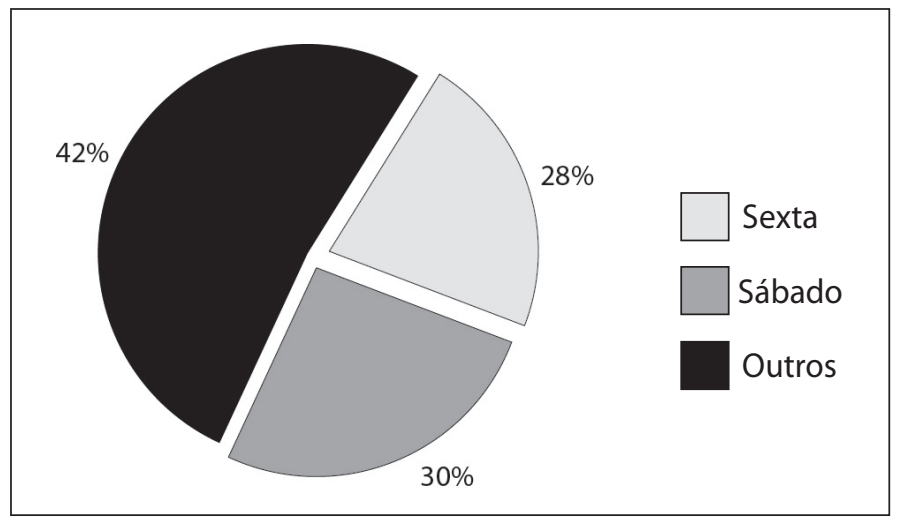

Figura 2. Dia da semana do acontecimento do evento.

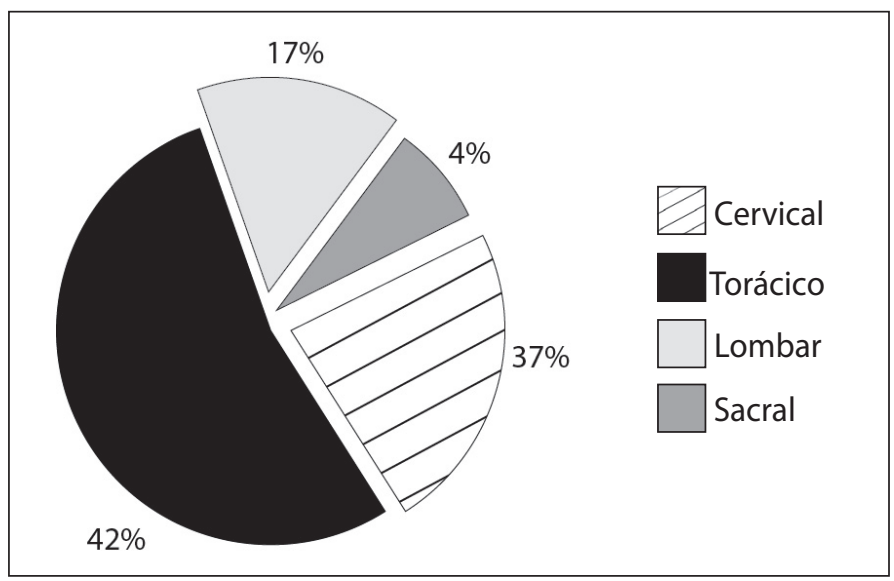

Figura 3. Segmento da coluna vertebral comprometido.

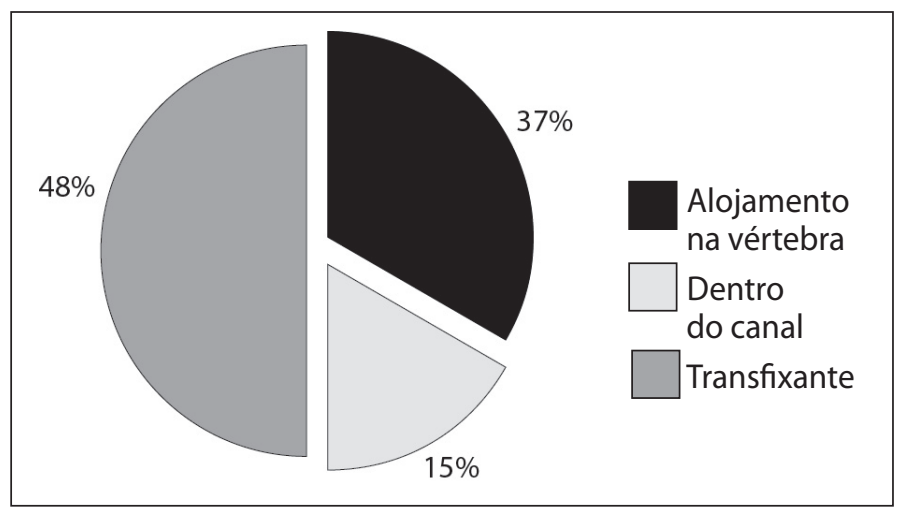

Figura 4. Localização do projétil de arma de fogo.

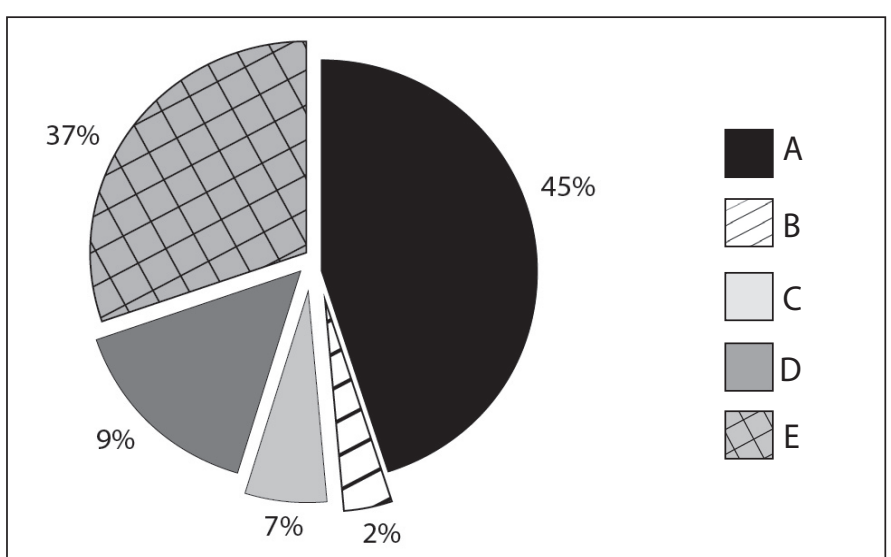

Figura 5. Classificação do déficit neurológico, de acordo com a escala da ASIA, dos pacientes vitimas de FPAF. 
Em 36 casos o tratamento foi conservador $(66,6 \%)$ e 18 casos foi cirúrgico $(33,4 \%)$. Laminectomia com debridamento foi realizada em 10 casos $(55,5 \%)$ e artrodese do nível por instabilidade em oito casos ( $44,5 \%)$.

Dez pacientes vieram a falecer (18,5\%) devido às consequências de lesão de vísceras abdominais / torácicas, sepse ou respiratórias por lesões medulares altas. A média de internamento foi de 17,5 dias. Nos casos de paraplegia ou leões de vísceras / pulmonares associadas, essa média alterou-se para 22,7 dias.

\section{DISCUSSÃO}

O atendimento de pacientes vítimas da violência urbana tem se tornado cada vez mais frequente nos centros hospitalares ${ }^{5}$. A incidência de pacientes jovens traz grandes prejuízos pessoais e materiais. A importância dos serviços de atendimento pré-hospitalar é grande porque a maioria destes pacientes, são trazidos aos centros por estes serviços. $\mathrm{O}$ atendimento destes pacientes deve ser multidisciplinar, uma vez que são muito frequentes as lesões associadas das e vísceras abdominais e torácicas, estruturas neurais, vasculares e cardio-pulmonare ${ }^{10}$. A literatura mostra que é mais frequente nos pacientes jovens, do sexo masculino e o nível torácico o mais acometido devido à sua extensão, dados que nosso estudo comprovam $^{1-5}$. O predomínio de lesões transfixantes também pôde ser observado pelo presente estudo com predomínio de lesões medulares completas. Uma das variáveis mais aceitas pela literatura como fator prognóstico é a apresentação clínica à admissão ${ }^{11}$. Lesões completas apresentam muito baixo potencial de recuperação enquanto que as incompletas e de cauda equina apresentam potencial de recuperação de $47 \%$ e $86 \%$ respectivamente ${ }^{12}$. De acordo com o trajeto que o projétil segue, inúmeras outras estruturas podem ser lesadas. O diagnóstico das lesões de vísceras toraco-abdominais em pacientes com lesão medular completa e, portanto, desprovidos de sensibilidade, é difícil e deve sempre ser suspeitado. Há um consenso na literatura de que o tratamento é conservador na maioria dos casos. As indicações cirúrgicas ainda geram controvérsias ${ }^{12}$. Estudos recentes demonstram que o prognóstico desses doentes não é diferente quando tratados conservadoramente. Benzel et al. ${ }^{11}$ mostraram que até $86 \%$ dos doentes com lesão incompleta têm melhora com a descompressão cirúrgica. Barros Filho et al. ${ }^{3}$ descreveram indicações para cirurgia em casos com entrada via posterior com fratura associada de lâmina causando compressão extrínseca. Waters e $\mathrm{Sie}^{2}$ relataram benefícios em descompressões medulares de T12 a L4, mas não há melhora neurológica significativa nos demais níveis. No entanto, Aarabi et al. ${ }^{13}$ concluíram que a cirurgia não melhorou o prognóstico dos pacientes, além de ter aumentado o número de complicações. Uma indicação cirúrgica é para lesões incompletas com déficit progressivo por compressão radiologicamente demonstrável, incluindo fragmentos ósseos, fragmentos da bala ou hematoma extradural compressivo. A principal abordagem nesses casos é a laminectomia para descompressão do canal ${ }^{14}$. Em alguns outros casos, a fixação de uma coluna instável ou a descompressão

\section{REFERÊNCIAS}

1. Benato ML, Zaninelli EM, Graells XS. Avaliação da incidência das lesões por arma de fogo da coluna vertebral. Coluna/Columna. 2007;6(3):155-61.

2. Waters $R L$, Sie $I H$. Spinal cord injuries from gunshot wounds to the spine. Clin Orthop Relat Res. 2003;(408):120-5.

3. Barros Filho TEP, Oliveira RP, Barros EK, Von Uhlendorff EF, lutaka AS. Ferimentos por arma de fogo na coluna vertebral: estudo epidemiológico. Coluna/Columna. 2002;1(2):83-7. - Azevedo Filho HR. Gunshot wounds to the spine: study of 246 patients. Arq Neuropsiquiatr. 2001;59(3):199-208.

5. Gonçalves AMT, Rosa LN, Savordelli CL, Bonin GL. Aspectos epidemiológicos da lesão medular traumática na área de referência do Hospital Estadual Mário Covas. Arq Med ABC. 2007;32(2):64-6.

6. Aryan HE, Amar AP, Ozgur BM, Levy ML. Gunshot wounds to the spine in adolescents. Neurosurgery. 2005;57(4):748-52.

7. Barros TEP, Zigler J, Aito S, Di Lorenzo N, Wing P. Gunshot wound to the spine. Spinal Cord. 2001;39(10):541-4.

8. Scuderi GJ, Vaccaro AR, Fitzhenry LN, Greenberg S, Eismont F. Long-term clinical manifestations of retained bullet fragments within the intervertebral disk space. . J Spinal Disord Tech. 2004;17(2):108-11.

9. Hanigan WC, Sloffer C. Nelson's wound: treatment of spinal cord injury in $19^{\text {th }}$ and early $20^{\text {th }}$ century military conflicts. Neurosurg Focus. 2004;16(1)1-12.

10. Medzon R, Rothenhaus T, Bono C, Grindlinger G, Rathlev NK. Stability of cervical spine after gunshot wounds to the head and neck. Spine (Phila Pa 1976). 2005;30(20):2274-9. radicular para alívio da dor também são procedimentos utilizados. Outras situações como intoxicação por chumbo, presença da bala perto de facetas ou liquido sinovial (com risco de corrosão da bala), ou fístulas liquóricas presentes também podem indicar cirurgia. Não há consenso também com relação ao tempo cirúrgico adequado. Cybulski et al. ${ }^{15}$ não encontraram diferença significativa quanto à meIhora neurológica em pacientes tratados com laminectomia em até 72 horas e aquelas realizadas após este período. Robertson e Simpson ${ }^{16}$ sugerem que nas lesões de cauda eqüina a cirurgia deve ser feita o mais breve possível. Segundo alguns autores, a cirurgia realizada muito precocemente poderia inclusive piorar o déficit neurológico pela manipulação de tecido neural edemaciado e a maior dificuldade de hemostasia ${ }^{14}$. Paciente tratados com cirurgia estão mais sujeitos à complicações como infecção, fístulas e instabilidade tardia ${ }^{12}$

O critério de instabilidade nos casos de ferimento por armas de fogo não está bem definido, uma vez que os projéteis determinam uma força direcional numa coluna estática e é menos passível de causar instabilidade, mesmo com lesão de duas ou três colunas de Denis ${ }^{12}$.

Complicações relatadas na literatura incluem infecção, fístula liquórica, intoxicação por chumbo, dor neuropática, artropatia neuropática de Charcot, migração do projétil entre outras ${ }^{12,17}$.

A profilaxia contra tétano é recomendada, bem como a antibioticoterapia. Esta varia de dois a três dias para lesões sem vísceras ocas até 7 a 14 dias quando houver lesão de vísceras ocas ou perfuração colônica. A decisão do tipo de antibiótico varia com o local do ferimento e lesões adicionais. Várias combinações podem ser usadas, sendo as mais comuns entre cefotaxima, gentamicina, clindamicina e penicilina. O índice de infecção para este tipo de lesão varia entre 7 a 22\%. A corticoterapia não é indicada mesmo nas lesões incompletas. Levy et al. ${ }^{18}$ revisaram 252 casos de lesões medulares por arma de fogo e concluiu que a metilprednisolona não alterou o prognóstico mesmo nas lesões incompletas. Heary et al. ${ }^{19}$ concluiram que não há melhora na recuperação de pacientes que fizeram uso da metilprednisolona. Tal fato provavelmente se deve ao mecanismo de lesão, que são distintos no trauma fechado e no perfurante. Traumas fechados raramente causam transecção da medula, estando a lesão mais associada ao infarto, isquemia, hipóxia e microhemorragias. No trauma perfurante, o mecanismo mais comum é laceração da medula.

A lesão medular pelos ferimentos de arma de fogo na coluna vertebral é atualmente uma entidade frequente e deve ser abordada de maneira multidisciplinar. O melhor tratamento ainda é discutível, mas sempre deve-se levar em consideração a intervenção cirúrgica para a descompressão medular e fusão vertebral, apesar de não causarem a reversão definitiva da lesão neurológica.

\section{CONCLUSÃO}

A maioria dos pacientes com lesão medular observada no estudo foi do o sexo masculino e o segmento vertebral mais acometido foi a coluna torácica, sendo a lesão medular completa a mais frequente.

11. Benzel EC, Hadden TA, Colemam JE. Civilian gunshot wounds to the spinal cord and cauda equina. Neurosurgery. 1987;20(2):281-5.

12. Cheryl A, Muszynsky KN. Surgical management of penetrating injuries to the spine. In: Schimidek Operative neurosurgical techniques. 3rd. Philadeplphia: Saunders, 1995. p. 1971-80.

13. Aarabi B, Alibaii $E$, Taghipur M, Kamgarpur A. Comparative study of functional recovery for surgically explored and conservatively managed spinal cord missile injuries. Neurosurgery. 1996;39(6):1133-40.

14. Simpson RK, Verger BH, Narayan RK. Treatment of acute penetrating injuries of the spine: a retrospective analysus. J Trauma. 1989;29(1):42-6.

15. Cybulski GR, Stone JL, Kant R. Outcome of laminectomy for civilian gunshot injuries of the terminal spinal cord and cauda equine: review of 88 cases. Neurosurgery. 1989;24(3):392-7.

16. Robertson DP, Simpson RK. Penetrating injuries restricted to the cauda equine: a retrospective review. Neurosurgery. 1992;31(2):265-9.

17. Flores LP, Nascimento Filho JS, Pereira Neto A, Suzuki K. Fatores prognósticos do trauma raquimedular por projétil de arma de fogo em pacientes submetidos a laminectomia. Arq Neuropsiquiatr. 1999;57(3B):836-42.

18. Levy ML, Gans W, Wijesinghe HS, SooHoo WE, Adkins RH, Stillerman CB. Use of methylprednisolone as an adjunct in management of patients qith penetrating spinal cord injury: outcome analysis. Neurosurgery. 1996; 39(6):1141-8.

19. Heary RF, Vaccaro AR, Mesa JJ, Northrup BE, Albert TJ, Balderston RA, et al. Steroids and gunshot wounds to the spine. Neurosurgery. 1997; 41(3):576-83. 